\title{
Development and Content Validation of Pruritus and Symptoms Assessment for Atopic Dermatitis (PSAAD) in Adolescents and Adults with Moderate-to- Severe AD
}

\author{
Rebecca Hall (D) - Mark G. Lebwohl • Andrew G. Bushmakin • Eric L. Simpson • Melinda J. Gooderham • \\ Andreas Wollenberg · Adam Gater · Jane R. Wells · Joseph C. Cappelleri · Ming-Ann Hsu • \\ Jocelyn Papacharalambous $\cdot$ Elena Peeva $\cdot$ Anna M. Tallman $\cdot$ Weidong Zhang $\cdot$ Linda Chen
}

Received: October 1, 2020 / Accepted: December 2, 2020 / Published online: December 26, 2020

(C) The Author(s) 2020, corrected publication 2021

\section{ABSTRACT}

Introduction: Most patient-reported outcome (PRO) instruments that measure atopic dermatitis (AD) symptoms do not have sufficient documented evidence of content validity to satisfy regulatory agency guidance for inclusion in product-labelling claims in the USA or Europe. The objective of this study was to develop a

Supplementary Information The online version contains supplementary material available at https:// doi.org/10.1007/s13555-020-00474-9.

R. Hall $(\bowtie) \cdot$ A. Gater · J. R. Wells Patient-Centered Outcomes, Adelphi Values, Adelphi Mill, Bollington, Cheshire, UK e-mail: rebecca.hall@adelphivalues.com

\section{G. Lebwohl}

Kimberly and Eric J. Waldman Department of Dermatology, Icahn School of Medicine at Mount Sinai, New York, NY, USA

A. G. Bushmakin

PIH Statistics, Pfizer Inc., Groton, CT, USA

E. L. Simpson

Department of Dermatology, Oregon Health and

Science University, Portland, OR, USA

\section{J. Gooderham}

SKiN Centre for Dermatology and Probity Medical Research, Peterborough, ON, Canada

\section{J. Gooderham}

Queen's University, Kingston, ON, Canada
PRO instrument in accordance with regulatory agency guidance to assess daily $\mathrm{AD}$ symptoms during the course of therapy and to establish its content validity and psychometric properties.

Methods: The Pruritus and Symptoms Assessment for Atopic Dermatitis (PSAAD) daily diary was developed based on qualitative interviews with US adolescents and adults with mild-tosevere AD. Content validity, test-retest reliability, internal consistency reliability, clinically important difference, clinically important responder, convergent validity, and knowngroup validity were evaluated using correlational and regression methods from phase $2 \mathrm{~b}$

\section{A. Wollenberg}

Department of Dermatology and Allergy, Ludwig-

Maximilians-Universität München, Munich,

Germany

J. C. Cappelleri

Global Biometrics and Data Management, Pfizer

Inc., Groton, CT, USA

M.-A. Hsu

PIH-Global Health and Value, Pfizer Inc., Groton, CT, USA

J. Papacharalambous · A. M. Tallman · L. Chen

Pfizer Inc., New York, NY, USA

E. Peeva

R\&D-Inflammation and Immunology, Pfizer Inc., Cambridge, MA, USA

W. Zhang

Pfizer Inc., Cambridge, MA, USA 
data from US adults with moderate-to-severe AD who were treated with abrocitinib.

Results: Patient interviews conducted with US adolescents and adults with mild-to-severe $\mathrm{AD}$ identified 11 relevant symptoms (itch, dryness, redness, flaking, discolouration, pain, bleeding, cracking, bumps, swelling, and weeping/oozing) for inclusion in the PSAAD instrument. All PSAAD psychometric parameters were acceptable based on phase $2 \mathrm{~b}$ data from US adults with moderate-to-severe AD. Convergent validity and known-group validity were confirmed by significant correlations between PSAAD and six other PRO measures $(r=0.24-0.91$, all $p \leq 0.01)$ and Dermatology Life Quality Index category $(p \leq 0.0001)$, respectively.

Conclusions: Evidence supports the PSAAD instrument validity, reliability, responsiveness and definitions of clinically important changes/ differences for adults with moderate-to-severe $\mathrm{AD}$.

Keywords: Atopic dermatitis; Daily diary; Eczema; Patient-reported outcomes; Pruritus

\section{Key Summary Points}

Most patient-reported outcome instruments that measure atopic dermatitis (AD) symptoms do not fulfil regulatory guidance for product-labelling claims.

The objective of this study was to develop a PRO instrument in accordance with regulatory agency guidance to assess daily $\mathrm{AD}$ symptoms during the course of therapy and to establish its content validity and psychometric properties.

Qualitative and quantitative evidence supports the validity, reliability and responsiveness of the Pruritus and Symptoms Assessment for Atopic Dermatitis (PSAAD) daily diary for assessing symptom severity and treatment response in adults with moderate-tosevere $\mathrm{AD}$.

\section{DIGITAL FEATURES}

This article is published with digital features, including a summary slide, to facilitate understanding of the article. To view digital features for this article go to https://doi.org/10.6084/ m9.figshare.13312178.

\section{INTRODUCTION}

Among skin diseases, atopic dermatitis (AD) is associated with a major burden of disease [1] and a significant proportion of patients with $\mathrm{AD}$ have inadequately controlled disease despite treatment [2]. Patient-reported severity of AD is often incongruous with physician-reported severity, with physicians frequently underestimating the severity of disease [3-5]. Patient-reported symptoms are among the set of core outcome measures recommended by the international Harmonising Outcome Measures for Eczema initiative [6, 7].

Most patient-reported outcome (PRO) instruments that measure $\mathrm{AD}$ symptoms do not provide a comprehensive assessment of all symptoms important to patients or do not have documented evidence of content validity (see Table S1 in Online Resource 1 for definitions of psychometric terms) that would be considered sufficient by the US Food and Drug Administration (FDA) or the European Medicines Agency (EMA) as a clinical trial endpoint to support product-labelling claims. This report details the development of a patient-reported symptom diary in accordance with FDA [8] and EMA [9] PRO guidance using qualitative interviews with adolescents and adults with mild-tosevere $A D$ and evaluation of its psychometric properties using data from a phase $2 b$ study in adults with moderate-to-severe AD [10].

\section{METHODS}

The PSAAD development was reviewed and approved by a centralised review board for conduct in the USA (Copernicus Group Independent Review Board; tracking number: ADE215-310). The data used for psychometric 
validation of the PSAAD were from a phase $2 b$ study (NCT027801670), which was also approved by institutional review boards at each study site [10]. All patients provided written informed consent and all research was conducted in accordance with the Helsinki Declaration of 1964 and its later amendments.

\section{PSAAD Content Development}

A review of the literature and of online patient blogs/forums (search of MEDLINE, Embase, and PsycINFO as well as Google Scholar and online patient blogs/forums relating to $\mathrm{AD}$ and dermatological conditions), physician input, and concept elicitation patient interviews were used to identify relevant $\mathrm{AD}$ symptoms and the language used by patients to talk about them (see Fig. S1 in Online Resource 1). Based on these findings, a draft 13-item daily diary was developed for completion via an electronic handheld device.

Thirty participants recruited from general practitioner or dermatologist offices in the USA were included in the concept elicitation interviews. Approximately ten interviews were conducted for each age group (12-14 years, $15-17$ years, and $\geq 18$ years) to achieve conceptual saturation (i.e. the point at which no new concepts are likely to be elicited in further interviews) [11-13]. Recruitment quotas were used to ensure adequate representation across sexes, physician- and patient-rated disease severity, racial and ethnic groups, and educational achievement (adults only).

To be eligible for interview, patients had to be aged $\geq 12$ years and have a clinical diagnosis of AD (using Hanifin and Rajka criteria [14]), affected percentage of body surface area (\%BSA) 2-40 (excluding scalp with $\% B S A \geq 2$ on body regions other than the palms and the soles), and physician-rated mild, moderate, or severe AD. Patients with contact or seborrhoeic dermatitis; discoid, gravitational/stasis, asteatotic or dyshidrotic eczema; psoriasis; or viral, fungal, or bacterial infection were excluded.

Patients participated in two semi-structured face-to-face interviews, each lasting approximately $1 \mathrm{~h}$. Interviewers were experienced in conducting interviews with adolescents and adults and were trained in the use of the interview guide and the electronic diary device. The first interview was designed to explore symptoms experienced by patients (i.e. concept elicitation) through open-ended questions, followed by more probing questions to explore concepts either not mentioned spontaneously or warranting further exploration/clarification. After the first interview, patients completed the draft 13-item daily diary at home once daily for 7 days using a supplied electronic device. The device included an alarm to remind patients to complete the diary each evening within the designated completion window. A second interview was then conducted to evaluate comprehension and relevance of diary content and user acceptability of the electronic instrument (i.e. cognitive debriefing). Interviews were audio recorded and transcribed verbatim for analysis. Interviews were conducted over two rounds. Updates made to the instrument based on first-round feedback were tested in the second round (Fig. S1 in Online Resource 1).

\section{PSAAD Psychometric Validation/ Quantitative Evaluation in a Phase $2 b$ Clinical Trial}

Psychometric evaluation of the PSAAD was performed using data from adults in the USA with moderate-to-severe $\mathrm{AD}$ included in a phase $2 b$ study of abrocitinib (NCT02780167) [10]. Accepted methods for psychometric and quantitative evaluation were applied [15-17]. Test-retest reliability was assessed using intraclass correlation coefficient (ICC; with a oneway random effects model), defined as betweenpatient variability divided by total variability (i.e. between-patient variability plus within-patient variability) [16] using pre-treatment data collected for $\geq 7$ days during the screening period. ICC values were considered acceptable if $\geq 0.70$ [18] and excellent if $>0.9$ [19]. Although patients completed the PSAAD daily, single measurements had acceptable test-retest reliability, so internal consistency reliability was evaluated using Cronbach's coefficient alpha and corrected item-to-total correlations based 
on data from days 1,1 (baseline), $8,15,29,43$, $57,85,92,99$, and 113. Acceptability criteria for Cronbach's coefficient alpha and corrected item-to-total correlations were $\geq 0.70 \quad$ [18] and $\geq 0.40$, respectively [20].

Convergent validity was assessed using Pearson correlation coefficients $(r)$ between PSAAD and other measures, including pruritus numeric rating scale (NRS; assesses the severity/ frequency of itching over the previous $24 \mathrm{~h}$ from no/never itching [0] to worst possible/always or constantly itching [10]), patient global assessment (PtGA; evaluates overall cutaneous disease at time of assessment on 5-point Likert scale ranging from clear [0] to severe [4]), patient global impression of severity (PGIS; daily 11-category scale to assess $\mathrm{AD}$ severity over the previous $24 \mathrm{~h}$, ranging from not present [0] to extremely severe [10]), patient global impression of change (PGIC; weekly 7-category scale to evaluate change in $\mathrm{AD}$ severity from baseline (ranging from much better [1] to much worse [7]), Dermatology Life Quality Index (DLQI), Patient-Oriented Eczema Measure (POEM), Investigator's Global Assessment (IGA), Eczema Area and Severity Index (EASI), $\% B S A$ and SCORing of AD (SCORAD). Correlation coefficients $\geq 0.40$ were considered supportive of convergent validity; those between 0.30 and 0.40 indicated no evidence for convergent or divergent validity, and those $<0.30$ indicated divergent validity [16]. Correlations between PSAAD and pruritus NRS, PtGA, IGA, EASI, \%BSA, or SCORAD were calculated using the average of daily scores from days $1,8,15$, $29,43,57$, and 85 . Correlation between PSAAD and PGIS was calculated using the average of daily scores from day 1 to day 88. Correlation between PSAAD and PGIC was based on the change from baseline in weekly average of daily PSAAD scores and weekly PGIC scores from week 1 to week 12. Correlation between PSAAD and POEM was based on weekly average of daily PSAAD scores and weekly POEM score for weeks $0,1,2,4,6,8$, and 12 .

PGIS and PGIC are anchors recommended by the FDA, along with relevant well-established clinical outcomes, to calculate a clinically meaningful change in a new patient-reported outcome [21]. A clinically important difference
(CID; difference between treatment groups considered clinically relevant) threshold in PSAAD total score was estimated by assessing the relationship between PSAAD total score and PGIS using a repeated-measures model and data from the 12-week double-blind part of the phase 2 b study (up to day 88). PGIS was assessed daily using an 11-category scale to assess AD severity over the previous $24 \mathrm{~h}$ (not present [0] to extremely severe [10]). Empirical research and historical precedent indicate that a 7-point Likert scale is preferred for important difference calculations [22, 23]. Based on this, CID was defined as the difference in mean PSAAD total score corresponding to a 1.7-point difference in PGIS (i.e. 10 divided by 6 , where 6 is the number of pairwise adjacent categories in PSAAD compared with that in PGIS). Sensitivity analyses for CID were performed using a repeated-measures model to estimate the relationship between PSAAD scores and PGIC and the relationship between PSAAD scores and POEM total scores (assuming that the CID of 3.4 points for POEM [24] would correspond to the CID for PSAAD). These relationships were analysed using PGIS, PGIC, and POEM total score each as a continuous anchor (which imposed a linear relationship between outcome and anchor) and as a categorical anchor (which did not impose any functional relationship between outcome and anchor).

Clinically important response (CIR; withinpatient change considered clinically relevant according to 'responder' criteria) threshold in PSAAD total score was examined with regard to the relationship between change in PSAAD and subject global impression of change (SGIC) by a repeated-measures model. SGIC is based on PGIC using the following algorithm: PGIC $\leq 3$, SGIC $=1$ (better); $\quad$ PGIC $=4, \quad$ SGIC $=0$ (the same); $\mathrm{PGIC} \geq 5$, SGIC $=-1$ (worse). Difference in change in mean PSAAD score corresponding to a 1-category difference in SGIC was used to define CIR. Standardised effect sizes of CID and CIR for PSAAD total score were obtained by dividing CID and CIR estimates by the standard deviation (SD) of baseline PSAAD total score. Criteria for the impact of an intervention in terms of effect sizes were: 0.2, 'small'; 0.5, 'medium'; 0.8, 'large' [17, 25]. 
With a repeated measures longitudinal model, known-group validity was determined by examining the relationship between PSAAD and DLQI, a dermatology-specific measure of health-related quality of life that is validated in dermatology clinical trials according to EMA standards [26], and calculating the mean difference in PSAAD between patients with 'no effect at all on patient's life' (DLQI $=0$ or 1 ) and those with at least a 'small effect on patient's life' (DLQI $\geq 2$ ).

\section{RESULTS}

\section{PSAAD Development/Qualitative Evaluation of Content Validity}

Iterative (repeated) concept elicitation and cognitive debriefing interviews were conducted with 30 adolescents and adults in the USA with mild-to-severe $\mathrm{AD}$ (round 1, $n=14$; round 2, $n=16$ ). Their disease characteristics were consistent with those of the overall adolescent and adult $\mathrm{AD}$ patient population in the USA and included the full range of AD severities and an adequate representation of lower education levels (Table S2 in Online Resource 1).

A review of the literature and patient forums/ blogs identified itch (pruritus), dryness (xerosis), redness (erythema), flaking, discolouration, pain (soreness, burning, stinging), bleeding, cracking, swelling/inflammation (oedema), weeping/oozing (fluid/exudate), tightness, and thickening as symptoms experienced by patients with $\mathrm{AD}$. Concept elicitation interviews identified the terminology used by patients for $\mathrm{AD}$ symptoms and confirmed the relevance of all but two of these symptoms to patient reporting (relevant: itch, dryness, redness, flaking, discolouration, pain, bleeding, cracking, swelling, fluid; not as relevant: tightness, thickening) and identified an additional symptom (bumps) (Fig. 1). Conceptual saturation was achieved across the concept elicitation interviews (Fig. 1).

Itch was by far the most relevant symptom, with all 30 patients reporting it spontaneously. Itch was also reported as the most frequent, severe, and bothersome symptom. Skin thickening and skin tightening were not considered important symptoms because they were rarely (if at all) mentioned by patients unless probed. Furthermore, more than half the patients did not report skin thickening or skin tightening items as relevant (57\% for each); therefore these symptoms were not included in the final PSAAD. All other symptoms, except for fluid (exudate), were reported by at least half the patients.

Most of the 11 symptoms included in the PSAAD were reported with similar frequency by adults and adolescents, except for fluid and cracking, which were reported slightly more frequently by adult patients. All 11 symptoms were reported across the spectrum of AD severities. Skin dryness, itching, and redness were reported by patients as the most frequent symptoms, whereas pain, weeping, itching, and bleeding were reported as the most bothersome.

Feedback during cognitive debriefing interviews indicated that instructions, items, and response options were consistently interpreted and appeared to be well understood by participants. Completion rates were good, and there were few skipped items or missing days; $57 \%$ of patients completed the diary every day during the 7-day period, and the mean number of completions was 6 . The majority of patients found the personalised alarm useful or essential to remind them to fill in the diary each day. Patients reported being able to successfully complete the daily diary using the electronic device; the mean time for daily completion was $2 \min 39 \mathrm{~s}$.

\section{PSAAD Instrument}

The final PSAAD is an 11-item instrument designed to provide a comprehensive assessment of symptom severity over the previous $24 \mathrm{~h}$ in adults (aged $\geq 18$ years) and adolescents (aged 12-17 years) with diagnoses of mild-to-severe AD (see www.pfizerpatientreportedoutcomes.com for further information). Each item of the PSAAD assesses the severity of a single symptom on an 11-point NRS, ranging from 0 (none) to 10 (extreme), and contributes equally to the PSAAD total score as depicted in the conceptual 


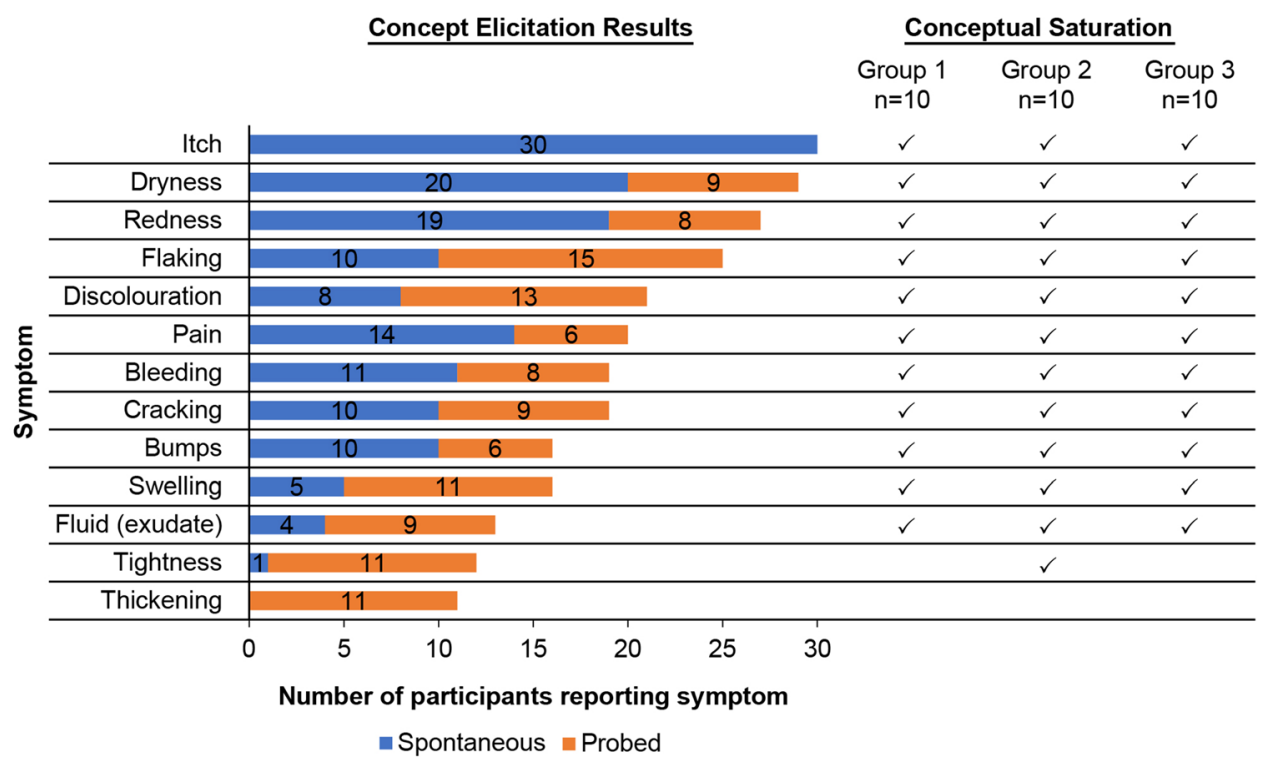

Fig. 1 Summary of concept elicitation and conceptual saturation results for atopic dermatitis symptoms. The number of spontaneous (blue) and probed (orange) reports of each symptom are displayed along with the group of

framework (Fig. 2). The PSAAD total score is calculated as the average of the responses to each of the 11 items, for a PSAAD total score range of 0 (none) to 10 (extreme).

\section{PSAAD Psychometric Validation/ Quantitative Evaluation}

The psychometric evaluation of the PSAAD was based on data from adult patients in the USA with moderate-to-severe AD who were enrolled in a phase $2 \mathrm{~b}$ study for abrocitinib, involving 12 weeks of treatment and a 4 -week follow-up period (Table S3, Online Resource 1); $81 \%$ of patients completed the PSAAD on $>70 \%$ of days in the phase $2 \mathrm{~b}$ study. Test-retest reliability of a single measurement was acceptable with ICC $>0.7$ (Table 1). Internal consistency reliability was excellent with Cronbach coefficient alpha $>0.9$ at every time point (Table 1; see Table S4 in Online Resource 1). Convergent validity was confirmed by substantial correlations in the expected direction between PSAAD and other measures (Table 2) ( $p \leq 0.01$ for all). concept elicitation transcripts with which each symptom was spontaneously mentioned (checkmarks) to assess conceptual saturation. Note: Interviews were divided into three equally sized groups (group 1, group 2, group 3 )

Based on anchors PCIS and PGIC, the CID and CIR of PSAAD total score were estimated to be 0.63 and 1.0 points, respectively, which represent approximately 'small' and 'medium' effect sizes of 0.28 and 0.45 (Table 1). The PGICand POEM-based estimates of CID (0.65 and 0.64 , respectively) were in agreement with the estimate based on PCIS. The close relationship demonstrated between PSAAD total score as a function of PGIS, PGIC or POEM total score as continuous and as categorical anchors supports the linearity assumption in the main CID model (Fig. 3).

A positive relationship between PSAAD and DLQI was evident (see Fig. S2 in Online Resource 1), with differences in PSAAD between groups with 'no effect at all on patient's life' (DLQI $=0$ or 1 ) and 'small to extremely large effect on patient's life' (DLQI $\geq 2$ ) all greater than the CID (0.63) and all statistically significant $(p \leq 0.0001)$ (Table 3). More severe symptoms according to PSAAD were associated with greater deficits in quality of life according to the DLQI, with DLQI total scores of 0-1 ('no effect'), 2-5 ('small effect'), 6-10 ('moderate effect'), 11-20 ('very large effect') and 21-30 ('extremely 


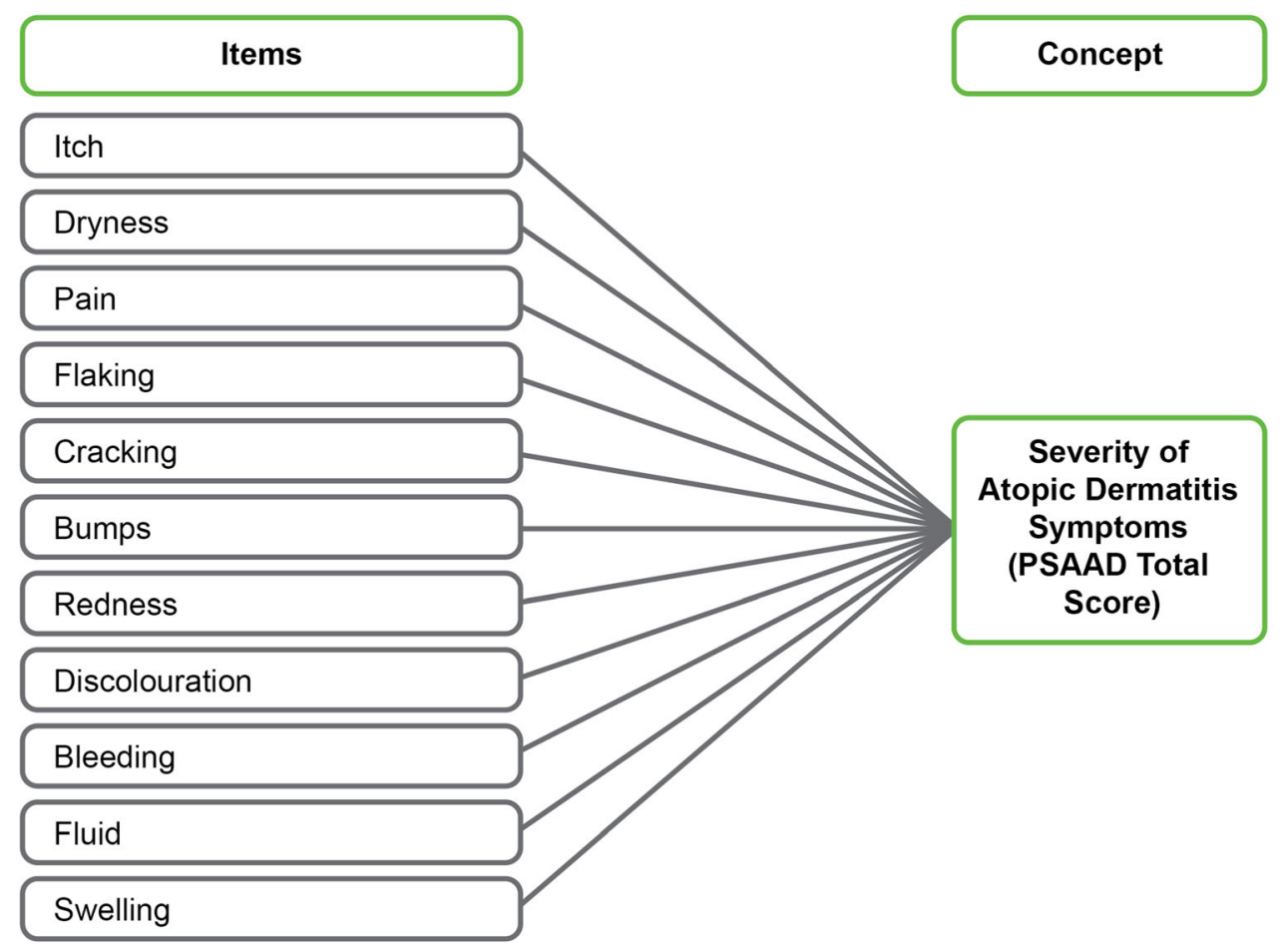

Fig. 2 PSAAD diary conceptual framework. $A D$ atopic dermatitis, PSAAD Pruritus and Symptoms Assessment for Atopic Dermatitis

Table 1 Psychometric validation parameters for the PSAAD diary

\begin{tabular}{lll}
\hline Parameter & Acceptability criteria & Actual \\
\hline Test-retest reliability & & 0.81 (for a single \\
Intraclass correlation coefficient & $>0.9$ excellent, & measurement) \\
& $0.7-0.9$ acceptable, $<0.7$ inadequate & $>0.9$ (every time point) \\
Internal consistency reliability & $\geq 0.70$ acceptable & $>0.5$ (every time point) \\
Cronbach coefficient alpha & $\geq 0.40$ acceptable & 0.63 \\
Corrected item-to- & - & 0.28 \\
total correlations & & 1.03 \\
CID & 0.80 large, 0.5 medium, 0.2 small & 0.45 \\
Effect size, SD units & - & \\
CIR & 0.80 large, 0.5 medium, 0.2 small & \\
Effect size, SD units & & \\
\hline
\end{tabular}

CID clinically important difference, CIR clinically important response, PSAAD Pruritus and Symptoms Assessment for Atopic Dermatitis, $S D$ standard deviation 
Table 2 Convergent validity: correlations between PSAAD diary and other measures

\begin{tabular}{cl}
\hline & $\begin{array}{l}\text { Pearson correlation coefficient with } \\
\text { PSAAD, } \boldsymbol{r}\end{array}$ \\
\hline $\begin{aligned} \text { Pruritus } \\
\text { NRS }^{\mathrm{a}}\end{aligned}$ & 0.82 \\
PtGA $^{\mathrm{a}}$ & 0.70 \\
PGIS $^{\mathrm{b}}$ & 0.91 \\
PGIC $^{\mathrm{c}}$ & 0.68 \\
DLQI $^{\mathrm{d}}$ & 0.67 \\
POEM $^{\mathrm{d}}$ & 0.82 \\
IGA $^{\mathrm{a}}$ & 0.38 \\
EASI $^{\mathrm{a}}$ & 0.37 \\
\%BSA $^{\mathrm{a}}$ & 0.24 \\
SCORAD $^{\mathrm{a}}$ & 0.60 \\
\hline
\end{tabular}

All correlations were calculated based on means of available data (see footnotes). Correlation coefficients $\geq 0.40$ were considered supportive of convergent validity, those between 0.30 and 0.40 indicated no evidence for convergent or divergent validity and those $<0.30$ indicated divergent validity [15] $p$ values $<0.01$ for all

$\% B S A$ percentage of body surface area, DLQI Dermatology Life Quality Index, EASI Eczema Area and Severity Index, $I G A$ Investigator's Global Assessment, $N R S$ numeric rating scale, PGIC patient global impression of change, PGIS Patient Global Impression of Severity, POEM PatientOriented Eczema Measure, PSAAD Pruritus and Symptoms Assessment for Atopic Dermatitis, PtGA Patient Global Assessment, SCORAD Scoring Atopic Dermatitis

a Average of daily scores for days 1, 8, 15, 29, 43, 57, and 85 for both variables

b Average of daily scores from day 1 to 88 for both variables

c Change from baseline in weekly average of daily PSAAD scores versus weekly PGIC scores from week 1 to 12

d Weekly average of daily PSAAD scores versus weekly POEM score for weeks $0,1,2,4,6,8$, and 12

large effect'), corresponding to PSAAD overall scores of approximately 2.6, 3.3, 4.2, 5.2 and 5.9, respectively (see Fig. S2 in Online Resource). This supports the clinical relevance of the changes observed and the known-group validity of the PSAAD.

\section{DISCUSSION}

Concept elicitation and conceptual saturation results indicate that the PSAAD captures all the symptoms of $\mathrm{AD}$ considered important by patients. Cognitive debriefing interviews confirmed comprehension and relevance of the instrument content among a diverse sample of adolescents and adults with $\mathrm{AD}$ in terms of age, sex, and physician-rated AD severity (mild to severe). Patient samples were ethnically and racially diverse across Black, White, multiracial, and other groups in both the qualitative and the quantitative phases. This ensures broad applicability of the measure.

Of note, this analysis defined both the between-group difference and the within-patient change considered to be clinically relevant (CID and CIR, respectively). Although many clinical trials use the former to evaluate treatment effects, which remains important, the FDA has been placing an emphasis on the latter because it represents a meaningful change from the patient perspective [21].

Unlike POEM and other more recently developed PROs (ADerm SS, Itch Numeric Rating Scale [v2.0], Skin Pain Numeric Rating Scale [v2.0b] and Peak Pruritus Numerical Rating Scale), the PSAAD provides a comprehensive assessment of $\mathrm{AD}$ symptom severity over the previous $24 \mathrm{~h}$ for all symptoms considered important by adults and adolescents with mildto-severe AD. Furthermore, PSAAD was developed to meet regulatory guidance and to be included in product-labelling claims in the USA and Europe. These results confirm previous research that itch is a central feature of $\mathrm{AD}$ from the patient perspective [28]. Itch was the only symptom reported by all 30 interviewees, all of whom reported it spontaneously, and it was also reported by interviewees as the most frequent, severe, and bothersome symptom. Skin dryness and redness were reported by almost all patients, with approximately two-thirds 
A

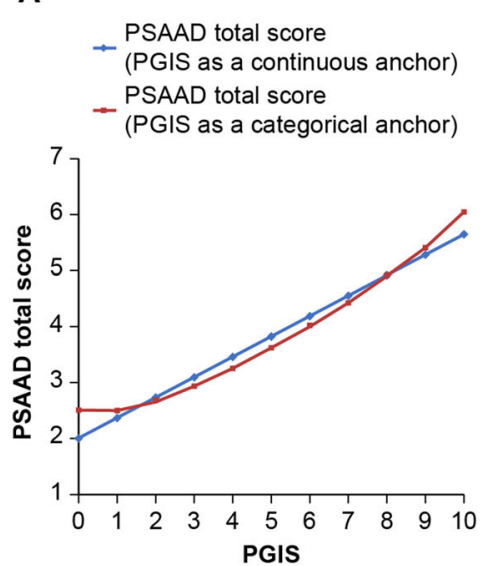

B

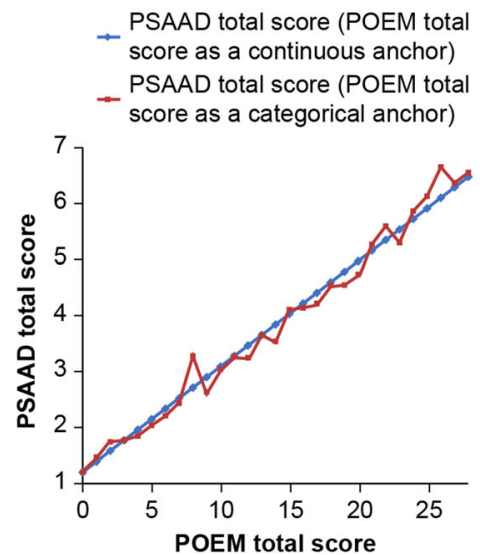

C

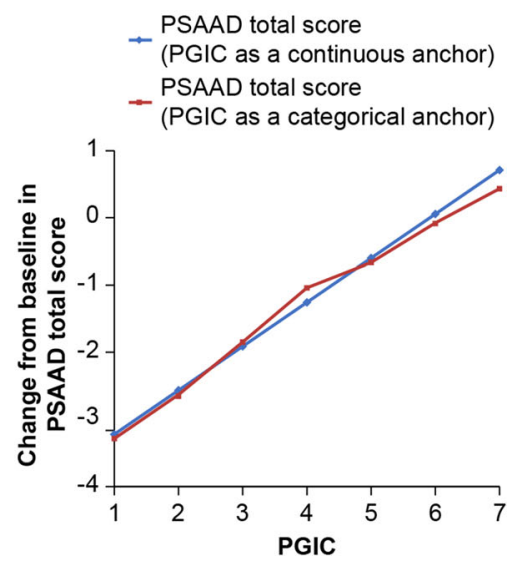

Fig. 3 Relationship between a PSAAD total score and PGIS, b PSAAD total score and POEM total score and c change from baseline in PSAAD total score and PGIC. $P G I C$ patient global impression of change, PGIS patient

Table 3 Known-group validity

\begin{tabular}{lll}
\hline DLQI range [27] & $\begin{array}{l}\text { Mean difference in } \\
\text { PSAAD total score } \\
\text { versus reference }\end{array}$ & $p$ value \\
\hline $\begin{array}{l}\text { 0-1: no effect at } \\
\text { all on patient's }\end{array}$ & Reference & N/A \\
life & \\
2-5: small effect & -0.7268 & $\leq 0.0001$ \\
on patient's life & & $\leq 0.0001$ \\
$\begin{array}{l}\text { 6-10: moderate } \\
\text { effect on }\end{array}$ & -1.6364 & \\
patient's life & & $\leq 0.0001$ \\
11-20: very large & -2.6757 & \\
effect on & & \\
patient's life & & \\
21-30: extremely & -3.3830 & \\
large effect on & & \\
patient's life & & \\
\hline
\end{tabular}

$D L Q I$ Dermatology Life Quality Index, $N / A$ not applicable, PSAAD Pruritus and Symptoms Assessment for Atopic Dermatitis

reporting them spontaneously. Although thickening is an important clinical feature associated with $\mathrm{AD}$ [29], it was only reported by global impression of severity, POEM Patient-Oriented Eczema Measure, PSAAD Pruritus and Symptoms Assessment for Atopic Dermatitis

patients when probed and was considered not relevant by a majority of patients. Therefore, thickening was not included in the final 11item PSAAD instrument.

By their nature, patient-reported AD symptoms such as itch are subjective; however, evidence from the qualitative interviews and the phase 2 study supports the reliability, content and construct validity, the definitions of clinically important changes, and the use of the PSAAD for assessing symptom severity in adults with moderate-to-severe $\mathrm{AD}$ in the USA. As expected, the PSAAD correlates well with POEM, SCORAD, and other measures of AD severity, which include a patient-reported subjective assessment of pruritus, but not as well with clinician-assessed objective measures such as EASI, IGA, and \%BSA, which do not. The lower correlations with EASI, IGA, and \%BSA may be indicative of divergent validity or lack of evidence to dismiss either convergent validity or divergent validity [16]. Furthermore, the relationship observed between PSAAD and DLQI confirms the substantial detrimental effects of pruritus and other AD symptoms on quality of life.

This study had some limitations. The PSAAD development population was relatively small and only included adults in the USA. While the sample size in this study was limited, similar- 
sized populations have been used for the development of other PRO qualitative research tools [11-13]. Additionally, the utility of the PSAAD was assessed in the context of a phase $2 b$ study that included a larger patient population, thereby providing a broader context for its use. Regarding the diversity of the population, to better understand the generalisability of the findings, future evaluation of the instrument should include a larger sample size, younger patients, and/or patients living outside the USA.

\section{CONCLUSION}

This study describes the development of the PSAAD, a daily PRO instrument to assess the severity of AD symptoms in clinical studies of novel treatments in accordance with regulatory agency guidance and evaluated its content validity and psychometric properties. The evidence presented herein supports the PSAAD instrument validity, reliability, responsiveness, and definitions of clinically important changes/ differences for adults with moderate-to-severe $\mathrm{AD}$, and confirms its suitability as an endpoint in clinical trials.

\section{ACKNOWLEDGEMENTS}

We thank the participants of the study.

Funding. This study was sponsored by Pfizer Inc., New York, NY, USA. Pfizer Inc. additionally funded the Rapid Service Fees.

Medical Writing Support. Medical writing support under the guidance of the authors was provided by Marianna Johnson, $\mathrm{PhD}$, and Jennifer C. Jaworski, MS, at ApotheCom, San Francisco, CA, USA, and was funded by Pfizer Inc., New York, NY, USA, in accordance with Good Publication Practice (GPP3) guidelines (Ann Intern Med. 2015;163:461-464).

Authorship. All named authors meet the International Committee of Medical Journal Editors (ICMJE) criteria for authorship for this article, take responsibility for the integrity of the work as a whole, and have given their approval for this version to be published.

Author Contributions. RH, MGL, AGB, AG, JRW, JCC, MH, JP, EP, AMT, WZ, and LC contributed to the conceptualization of the study. RH, AGB, AW, AG, JRW, JCC, MH, EP, WZ, and LC performed or supported the formal analysis. All authors (RH, MGL, AGB, ELS, MJG, AW, AG, JRW, JCC, MH, JP, EP,AMT, WZ, and LC) contributed to the data interpretation, critically reviewed the manuscript and approved the submitted version.

Disclosures. Rebecca Hall, Adam Gater, and Jane R. Wells are employees of Adelphi Values, which has received payment from Pfizer Inc. for conducting the instrument development research activities described in this paper. Mark G. Lebwohl is an employee of Mount Sinai, which receives research funds from Pfizer Inc., AbbVie, Bausch Health (Valeant), Boehringer Ingelheim, Celgene, Eli Lilly, Incyte, Janssen/ Johnson \& Johnson, LEO Pharma, Medimmune/AstraZeneca, Novartis, SCIderm, UCB, and Vidac Pharma. He is also a consultant for Allergan, Almirall (Aqua Pharmaceuticals), Boehringer Ingelheim, Dr. Reddy's Laboratory (Promius), LEO Pharma, Menlo Therapeutics, and Verrica. He is also a member of the journal's Editorial Board. Andrew G. Bushmakin Joseph C. Cappelleri, Ming-Ann Hsu, and Elena Peeva are employees and stockholders of Pfizer Inc. Eric L. Simpson has received grants, personal fees, and/or nonfinancial support from Pfizer Inc., AbbVie, Celgene, Eli Lilly, Galderma, GSK, LEO Pharma, Menlo Therapeutics, Novartis, Regeneron, Tioga Pharmaceuticals, and Vanda Pharmaceuticals. Melinda J. Gooderham has received grants, personal fees, and/or nonfinancial support from Pfizer Inc., AbbVie, Akros Pharma, Amgen, Arcutis, Bausch Health (Valeant), Boehringer Ingelheim, Bristol-Myers Squibb, Celgene, Coherus, Dermira, Eli Lilly, Galderma, Incyte, Janssen, Kyowa Kirin, LEO Pharma, MedImmune, Merck, Novartis, Regeneron, Roche, Sanofi Genzyme, Sun Pharma, and UCB. Andreas Wollenberg has received grants, personal fees, and/or nonfinancial support from Pfizer Inc., Almirall, Anacor, Astellas, Beiersdorf, 
Bioderma, Celgene, Chugai, Galderma, Hans Karrer, L'Oréal, LEO Pharma, Meda, MedImmune, Merck, Novartis, Pierre Fabre, Regeneron, and Sanofi. Jocelyn Papacharalambous, Anna M. Tallman, Weidong Zhang and Linda Chen were employees and stockholders of Pfizer Inc. at the time of this research.

Compliance with Ethics Guidelines. The PSAAD development was reviewed and approved by a centralized review board for conduct in the USA (Copernicus Group Independent Review Board; tracking number: ADE215-310). The data used for psychometric validation of the PSAAD was from a phase $2 b$ study (NCT027801670), which was also approved by institutional review boards at each study site [10]. All patients provided written informed consent and all research was conducted in accordance with the Helsinki Declaration of 1964 and its later amendments.

Data Availability. The datasets generated during and/or analyzed during the current study are available from the corresponding author on reasonable request. Upon request, and subject to certain criteria, conditions and exceptions (see https://www.pfizer.com/ science/clinical-trials/trial-data-and-results for more information), Pfizer will provide access to individual de-identified participant data from Pfizer-sponsored global interventional clinical studies conducted for medicines, vaccines and medical devices (1) for indications that have been approved in the US and/or EU or (2) in programs that have been terminated (ie development for all indications has been discontinued). Pfizer will also consider requests for the protocol, data dictionary and statistical analysis plan. Data may be requested from Pfizer trials 24 months after study completion. The deidentified participant data will be made available to researchers whose proposals meet the research criteria and other conditions, and for which an exception does not apply, via a secure portal. To gain access, data requestors must enter into a data access agreement with Pfizer.

Open Access. This article is licensed under a Creative Commons Attribution-
NonCommercial 4.0 International License, which permits any non-commercial use, sharing, adaptation, distribution and reproduction in any medium or format, as long as you give appropriate credit to the original author(s) and the source, provide a link to the Creative Commons licence, and indicate if changes were made. The images or other third party material in this article are included in the article's Creative Commons licence, unless indicated otherwise in a credit line to the material. If material is not included in the article's Creative Commons licence and your intended use is not permitted by statutory regulation or exceeds the permitted use, you will need to obtain permission directly from the copyright holder. To view a copy of this licence, visit http:// creativecommons.org/licenses/by-nc/4.0/.

\section{REFERENCES}

1. Hay RJ, Johns NE, Williams HC, et al. The global burden of skin disease in 2010: an analysis of the prevalence and impact of skin conditions. J Invest Dermatol. 2014;134:1527-34.

2. Wei W, Anderson P, Gadkari A, et al. Extent and consequences of inadequate disease control among adults with a history of moderate to severe atopic dermatitis. J Dermatol. 2018;45:150-7.

3. Wei W, Anderson P, Gadkari A, et al. Discordance between physician- and patient-reported disease severity in adults with atopic dermatitis: a US crosssectional survey. Am J Clin Dermatol. 2017;18: 825-35.

4. Magin PJ, Pond CD, Smith WT, et al. Correlation and agreement of self-assessed and objective skin disease severity in a cross-sectional study of patients with acne, psoriasis, and atopic eczema. Int J Dermatol. 2011;50:1486-90.

5. Torrelo A, Ortiz J, Alomar A, et al. Atopic dermatitis: impact on quality of life and patients' attitudes toward its management. Eur J Dermatol. 2012;22: 97-105.

6. Chalmers JR, Schmitt J, Apfelbacher C, et al. Report from the third international consensus meeting to harmonise core outcome measures for atopic eczema/dermatitis clinical trials (HOME). Br J Dermatol. 2014;171:1318-25. 
7. Schmitt J, Spuls P, Boers M, et al. Towards global consensus on outcome measures for atopic eczema research: results of the HOME II meeting. Allergy. 2012;67:1111-7.

8. US Department of Health and Human Services. Guidance for Industry: patient-reported outcome measures: use in medical product development to support labeling claims. 2020. https://www.fda. gov/downloads/drugs/guidances/ucm193282.pdf. Accessed 17 Jul 2020.

9. European Medicines Agency. Reflection paper on the regulatory guidance for the use of health-related quality of life (HRQL) measures in the evaluation of medicinal products. 2020. https://www.ema. europa.eu/documents/scientific-guideline/reflectionpaper-regulatory-guidance-use-healthrelated-qualitylife-hrql-measures-evaluation_en.pdf. Accessed 17 Jul 2020.

10. Gooderham MJ, Forman SB, Bissonnette R, et al. Efficacy and safety of oral Janus kinase 1 inhibitor abrocitinib for patients with atopic dermatitis: a phase 2 randomized clinical trial. JAMA Dermatol. 2019;155:1371-9.

11. Guest G, Bunce A, Johnson L. How many interviews are enough? Field Methods. 2016;18:59-82.

12. Francis JJ, Johnston M, Robertson C, et al. What is an adequate sample size? Operationalising data saturation for theory-based interview studies. Psychol Health. 2010;25:1229-45.

13. Turner-Bowker DM, Lamoureux RE, Stokes J, et al. Informing a priori sample size estimation in qualitative concept elicitation interview studies for clinical outcome assessment instrument development. Value Health. 2018;21:839-42.

14. Hanifin JM, Rajka G. Diagnostic features of atopic dermatitis. Acta Derm Venereol. 1980;60:44-7.

15. US Department of Health for Drug and Human Services. Guidance for Industry and FDA Staff: qualification process for drug development tools. 2020 . https://www.fda.gov/downloads/drugs/ guidances/ucm230597.pdf. Accessed 17 Jul 2020.

16. Cappelleri J, Zou K, Bushmakin A, et al. Patientreported outcomes: measurement, implementation and interpretation. Boca Raton: Chapman \& Hall/ CRC Press; 2013.

17. Cappelleri JC, Bushmakin AG. Interpretation of patient-reported outcomes. Stat Methods Med Res. 2014;23:460-83.
18. Chassany O, Sagnier $\mathrm{P}$, Marquis $\mathrm{P}$, et al. Patientreported outcomes: the example of health-related quality of life-a European guidance document for the improved integration of health-related quality of life assessment in the drug regulatory process. Drug Info J. 2016;36:209-38.

19. Koo TK, Li MY. A guideline of selecting and reporting intraclass correlation coefficients for reliability research. J Chiropr Med. 2016;15:155-63.

20. Ware JE Jr, Gandek B. Methods for testing data quality, scaling assumptions, and reliability: the IQOLA Project approach. International Quality of Life Assessment. J Clin Epidemiol. 1998;51:945-52.

21. US Food and Drug Administration. Discussion Document for Patient-Focused Drug Development Guidance Public Workshop on Guidance 3: select, develop or modify fit-for-purpose clinical outcomes assessments. Workshop date. 2018. https://www. fda.gov/media/116277/download. Accessed $17 \mathrm{Jul}$ 2020 .

22. Norman GR, Sloan JA, Wyrwich KW. Interpretation of changes in health-related quality of life: the remarkable universality of half a standard deviation. Med Care. 2003;41:582-92.

23. Norman GR, Sloan JA, Wyrwich KW. The truly remarkable universality of half a standard deviation: confirmation through another look. Expert Rev Pharmacoecon Outcomes Res. 2004;4:581-5.

24. Schram ME, Spuls PI, Leeflang MM, et al. EASI, (objective) SCORAD and POEM for atopic eczema: responsiveness and minimal clinically important difference. Allergy. 2012;67:99-106.

25. Cohen J. Statistical power analysis for the behavioral sciences. 2nd ed. Hillsdale: Lawrence Erlbaum Associates; 1988.

26. European Medicines Agency. Guideline on clinical investigation of medicinal products indicated for the treatment of psoriasis. 2020. https://www.ema. europa.eu/en/documents/scientific-guideline/guide line-clinical-investigation-medicinal-productsindicated-treatment-psoriasis_en.pdf. Accessed 17 Jul 2020.

27. Finlay AY, Khan GK. Dermatology Life Quality Index (DLQI) - a simple practical measure for routine clinical use. Clin Exp Dermatol. 1994;19: 210-6.

28. Charman CR, Venn AJ, Williams HC. The patientoriented eczema measure: development and initial validation of a new tool for measuring atopic 
eczema severity from the patients' perspective. Arch Dermatol. 2004;140:1513-9.

29. Eichenfield LF, Tom WL, Chamlin SL, et al. Guidelines of care for the management of atopic dermatitis: section 1. Diagnosis and assessment of atopic dermatitis. J Am Acad Dermatol. 2014;70: 338-51. 